MITSUBISHI ELECTRIC RESEARCH LABORATORIES

http://www.merl.com

\title{
A Non Parametric Approach for Modeling Interferometric SAR Imagery and Applications
}

\author{
Kuntal Sengupta, Prabir Burman
}

TR2008-052 August 2008

\begin{abstract}
In this paper, we present a non parametric modeling for phase maps of interferometric SAR. Cosine and Sine projections maps are generated from the SAR phase map, and each of them are individually modeled by fitting 2D basis functions. The coefficients of these basis functions describe a "smoothed" version of the original phase map. Several applications can be derived from this noise filtered phase map: better phase unwrapping and SAR image compression are two of the applications that we will be discussing in the paper. The approach can be extended to other imaging domains that involve large maps of directional or phase data, such as modeling of phase MRI images, modeling of wind directions in meteorological data, etc.
\end{abstract}

WACV 2008

This work may not be copied or reproduced in whole or in part for any commercial purpose. Permission to copy in whole or in part without payment of fee is granted for nonprofit educational and research purposes provided that all such whole or partial copies include the following: a notice that such copying is by permission of Mitsubishi Electric Research Laboratories, Inc.; an acknowledgment of the authors and individual contributions to the work; and all applicable portions of the copyright notice. Copying, reproduction, or republishing for any other purpose shall require a license with payment of fee to Mitsubishi Electric Research Laboratories, Inc. All rights reserved.

Copyright (C) Mitsubishi Electric Research Laboratories, Inc., 2008

201 Broadway, Cambridge, Massachusetts 02139 



\section{A non parametric approach for modeling interferometric SAR imagery and applications}

\author{
Kuntal Sengupta \\ MERL \\ sengupta@merl.com
}

\begin{abstract}
In this paper, we present a non parametric modeling for phase maps of interferometric SAR. Cosine and Sine projections maps are generated from the SAR phase map, and each of them are individually modeled by fitting $2 \mathrm{D}$ basis functions. The coefficients of these basis functions describe a "smoothed" version of the original phase map. Several applications can be derived from this noise filtered phase map: better phase unwrapping and SAR image compression are to of the applications that we will be discussing in the paper. The approach can be extended to other imaging domains that involve large maps of directional or phase data, such as modeling of phase MRI images, modeling of wind directions in meteorological data, etc.
\end{abstract}

\section{Introduction}

Synthetic aperture radar (SAR) involves imaging of the Earth by processing microwave backscattering data collected along the flight path of an aircraft or a spacecraft [1-2]. The data collection leads to highresolution images of the local complex ground reflectivity. Starting from two SAR images obtained from different flight paths, these images are first registered and a product image is computed by multiplying the first image with the complex conjugate of the second image. The phase of the product image is the SAR interferogram. Note that the phase is limited between $-\pi$ and $\pi$. The inherent circular nature of the data leads to the "artificial" discontinuities in the map. This "wrapped" phase map needs to be processed further.

Unwrapping the phase map leads to digital elevation model of the terrain [3]. Unwrapping involves the removal of the phase hops of more than $\pi$ between neighboring pixels, by adding or subtracting multiples of $2 \pi$. The assumption here is that the underlying function is a smooth function. However, in practical situations, the 2D phase is corrupted by noise. For example, temporal and baseline decorrelation introduces local inconsistencies

\author{
Prabir Burman \\ UC Davis \\ pburman@ucdavis.edu
}

in the data called the residues. 2D phase unwrapping algorithms are highly sensitive to residues, especially if these residues introduce local singularities in the map [4]. For example, given a pixel, if we walk around its eight neighbors, with this pixel in the center, the angle values should sum up to approximately zero. If the sum is close to a non zero multiple of $\pi$, this implies that the data has a singularity, and the map cannot be unwrapped. This fact is used widely in the fingerprint literature to find cores and deltas in the orientation field computed from the fingerprint image. Formally, this is called as the Poincare index method in locating feature points in fingerprint images [5].

Having noted that the noise sensitivity of 2D phase unwrapping algorithms is a critical factor in obtaining the digital elevation map, we need to devise techniques for noise filtering, or smoothing of the data. Most techniques reported in the literature treat the problem as an image denoising problem, and design filters to address the issue. One other application domain that we wish to address here is the compression of angle maps. By using a non parametric model fitting approach, we address both the problems of smoothing 2D phase maps as well as compression. There are two main contributions of the paper:

- we present an unique model based smooth representation of SAR phase maps

- we present a method of filtering noisy SAR phase maps.

To our knowledge, not much prior literature exists in the area of modeling/coding of 2D phase maps. The phase image datasets are often treated like normal intensity images, and compression algorithms such as wavelets or DCT are used. Our non parametric approach is an extension of our prior work in [7], and the algorithm/code developed there has been applied to solve this problem. Outside the domain of SAR imagery, there are several other applications where compression of large databases of angle maps are 
considered important. Three dimensional phase MRI, meteorological data with wind direction are a few of these examples.

\section{A Non Parametric Approach to Estimating the Phase Map Model in 1D}

A phase map can be fully represented by a discrete matrix whose elements represent the direction pointed to by a hypothetical vector planted at the corresponding pixel. To avoid the difficult problem created by orientation discontinuity, we create two distinct maps, the cosine and the sine map of the phase [7]. At the location $(i, j)$, if the phase value is $\Theta(i, j)$, then define the two maps $S$ and $C$ as follows:

$$
\begin{aligned}
& S(i, j)=\cos (\Theta(i, j)) \text { and } \\
& C(i, j)=\sin (\Theta(i, j)) .
\end{aligned}
$$

For ease of explanation, we would consider the discussion on the 1D case initially. For the sine curve (map), the noise model is:

$$
S\left(x_{i}\right)=Z\left(x_{i}\right)+\varepsilon\left(x_{i}\right) .
$$

In the equation above, $Z$ is the underlying (true) curve, and $\varepsilon$ is the noise at the location $x_{i}$, respectively. Assume $i=1,2, . . n$. Following [7], we model the underlying curve as a summation of basis splines as shown below:

$$
Z\left(x_{i}\right)=\boldsymbol{\psi}^{T}\left(x_{i}\right) \boldsymbol{\theta} .
$$

Here, $\psi\left(x_{i},\right)$ is the a vector of basis function values at the location $x_{i}$. For example, if we consider $m$ basis functions, then $\psi$ is $m \times 1$. Also, $\boldsymbol{\theta}$ is the weight associated with each basis function, and its dimension is $1 \times m$.

For a chosen set of basis functions, and our observation values $\mathrm{S}\left(x_{i}\right)$, we need to estimate the weights associated with these functions. For these, we use the Minimum Mean Squared approach. The cost function defined is:

$$
Q(\boldsymbol{\theta})=\frac{1}{n} \sum_{i}\left(S\left(x_{i}\right)-\boldsymbol{\psi}^{T}\left(x_{i}\right) \boldsymbol{\theta}\right)^{2} .
$$

To solve the above equation for the weight parameters, we construct two matrices as follows: and

$$
\mathbf{A}=\frac{1}{\mathrm{n}} \sum_{i} \boldsymbol{\psi}\left(x_{i}\right) \boldsymbol{\psi}^{T}\left(x_{i}\right)
$$

$$
\mathbf{B}=\frac{1}{n}\left(\sum_{i} S\left(x_{i}\right) \boldsymbol{\psi}^{T}\left(x_{i}\right)\right) .
$$

Note that $\mathbf{A}$ is a $m \times m$ matrix, and $\mathbf{B}$ is $m \times 1$ matrix. Next, we compute the eigenvector as follow:

$$
\Gamma=E I G E N_{-} \operatorname{VECTOR}\left(\mathbf{B} \mathbf{A}^{-1} \mathbf{B}^{T}\right) .
$$

The eigenvector considered above is the one that is associated with the largest eigenvalue. The estimated weight matrix is computed as:

$$
\hat{\boldsymbol{\theta}}=\mathbf{A}^{-\mathbf{1}} \mathbf{B}^{\mathrm{T}} \Gamma \text {. }
$$

The smooth sine projection of the phase map is:

$$
\widehat{S}(x)=\widehat{\boldsymbol{\theta}} \bullet \boldsymbol{\psi}(x) .
$$

The above process is repeated for the cosine map too, and similar weights of the spline function are determined.

\section{Choosing the Basis Functions and extension to $2 \mathrm{D}$}

Inspired by the Fourier Expansion work on fingerprint orientation map in [6], we choose $(2 \mathrm{~K}+1)$ the basis functions as below:

$$
\boldsymbol{\psi}(x)=\left\{\begin{array}{c}
1 / 2 \quad i=0 \\
\cos m v x \quad i=2 m-1 ; m=1,2, . ., K \\
\sin m v x \quad i=2 m ; m=1,2, \ldots, K
\end{array}\right.
$$

In the above equation, $v=\pi / n$. One nice property to note is that the basis functions are orthogonal, hence $A$ is a diagonal matrix. This makes the inversion function computation in Eq. [5] very trivial while computing the weight function. Other basis functions such as basis spline functions can be considered too, but for the computational efficiency, one can orthogonalize the basis set using the $\mathrm{QR}$ factorization technique. 

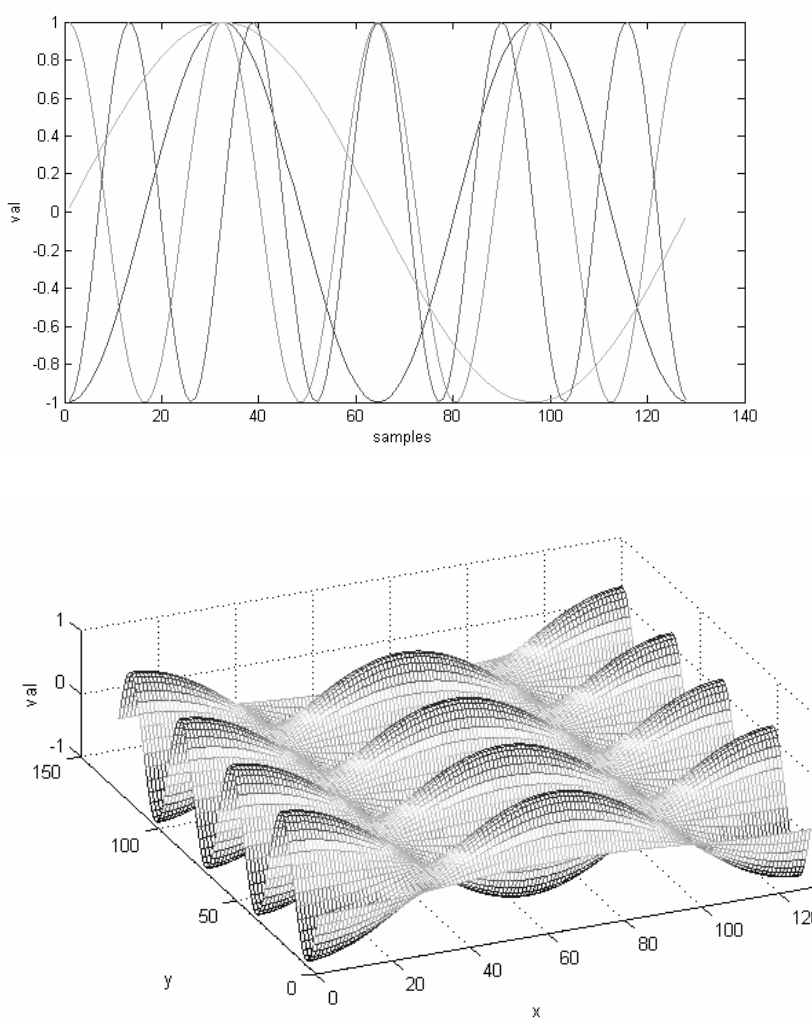

Figure 1: Plot of a few sample orthogonal 1D basis functions selected for the experiments here (top), and a mesh plot of the $2 \mathrm{D}$ basis function (bottom).

The extension of the technique described in Section 2 is straightforward. We now consider the tensor product of the basis functions in dimension $x$ and dimension $y$. The rest of the mathematics is almost similar, with the difference that we have $n_{x} \times n_{y}$ observations to deal with.Also, assuming that there are $(2 \mathrm{Kx}+1) 1 \mathrm{D}$ functions for the $\mathrm{x}$ dimension and $(2 \mathrm{Ky}+1)$ 1D functions for the $\mathrm{y}$ dimension, the weight vector has $(2 \mathrm{Kx}+1)(2 \mathrm{Ky}+1)$ elements or coefficients in it. A few example 1D and 2D basis functions are illustrated in Figure 1.

\section{Representation of the map: Quantization and Error Map}

As shown in Figure 2, the $(2 \mathrm{Kx}+1)(2 \mathrm{Ky}+1)$ coefficients of the basis functions corresponding to the estimated sine and the cosine maps, respectively, essentially code the phase map. Each of these coefficients are quantized and coded in " $\mathrm{N}$ " bits.
Thus, if we have $2(2 \mathrm{Kx}+1)(2 \mathrm{Ky}+1)$ coefficients modeling the cosine and the sine maps, the total storage requirements are $2 \mathrm{~N}(2 \mathrm{Kx}+1)(2 \mathrm{Ky}+1)$. Once these coefficients are known, the estimated cosine and sine maps are computed and the original phase map are estimated. The estimated phase map is smooth, and the error map is computed by subtracting the original map from the estimated map. The subtraction has to consider the wrap around issue. Keeping the circular nature data in mind, one can generate the error map, which is always bounded between $-\pi$ and $\pi$. This map can be further compressed using a standard compression technique, such as DCT based method or wavelet based methods. Since the dynamic range of the error map is generally low, a fewer number of bytes can easily code this map. The model based technique coupled with the error map helps us in achieving high compression ratio. Also, the resulting estimated phase map using the dưal strategy is noise filtered and can be unwrapped effectively.

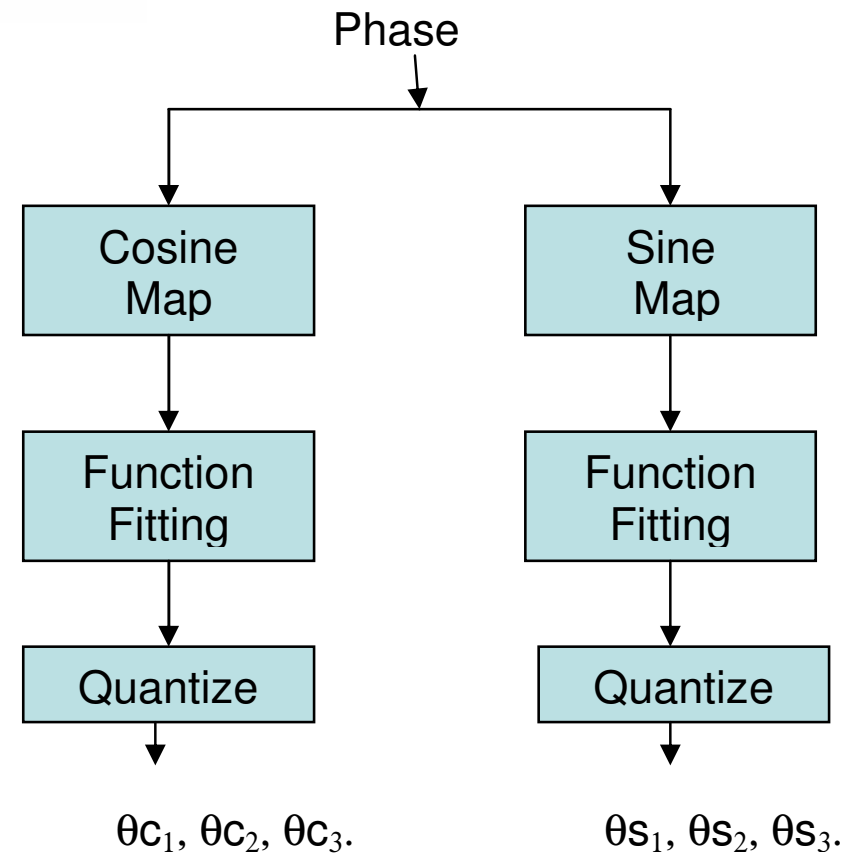

Figure 2: The model fitting approach for angle maps. 


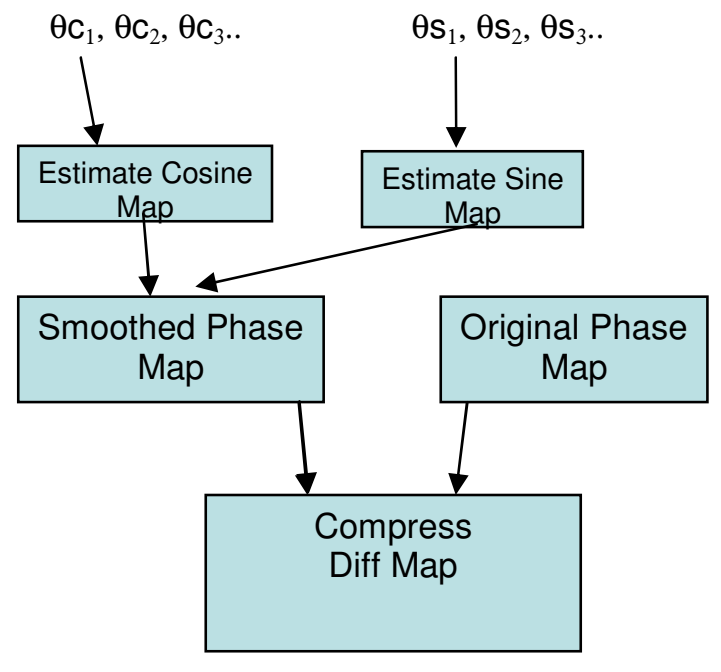

Figure 3: Generation of the difference map while considering the circular nature of the data.

\section{Experimental Results: Compression}

We obtained interferometric SAR images from the STAR Lab of Stanford University. The SAR image corresponded to an area around Mt. Etna. The phase map is computed from the raw SAR imagery using the software package ("snaphu")[8].

To perform our experiments, we divided the region into several blocks of $128 \times 128$ pixels. For each such block, we modeled the phase map using the non parametric method described in Section 2 of this paper. For our experiments, we used a total of 121 (11 functions in each dimension) 2D basis functions. The 242 coefficients obtained were coded using 1 byte each. The error map generated was coded using a standard DCT coding scheme, where each of the $128 \times 128$ blocks were subdivided into $8 \times 8$ blocks for DCT computation and coding. For a given byte allocation for the entire $128 \times 128$ block, whatever budget is left after using up the quota for the 242 (model) coefficients were used to code the DCT coefficients. In Figure 4, we illustrate one such patch of the SAR phase image. For illustration purposes, the phase value between 0 and $2 \pi$ is quantized between 0 and 255. The noise in the computed phase (from raw SAR data) is quite apparent in the image.

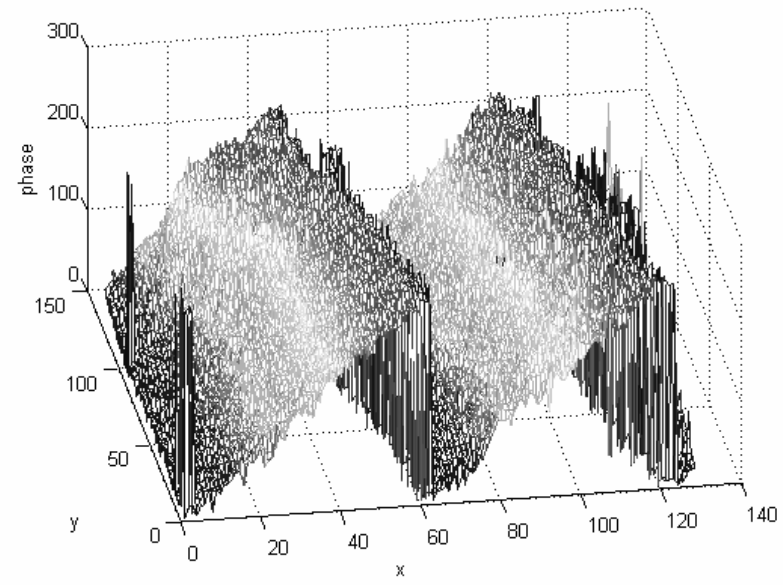

Figure 4: An example input phase map (note that the value of the phase has been quantized between 0 and 255).

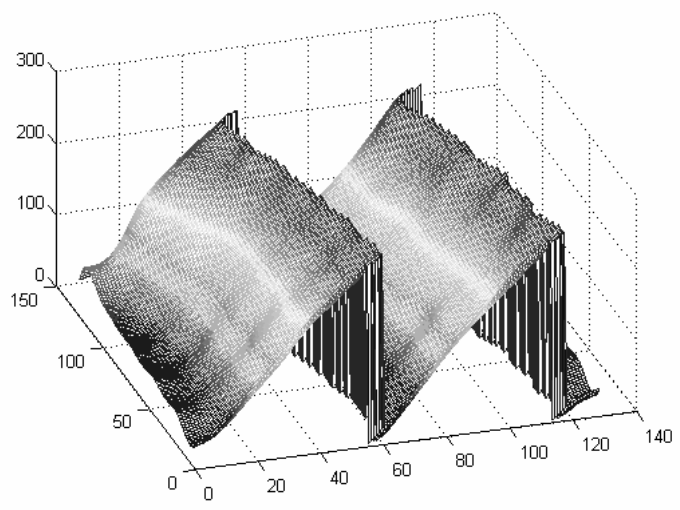

Figure 5: The modeled phase map.

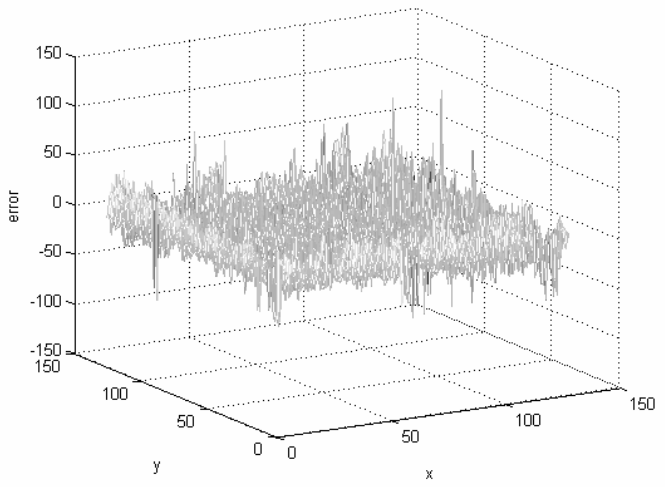

Figure 6: The error in the phase map modeling, which is coded using conventional means. 


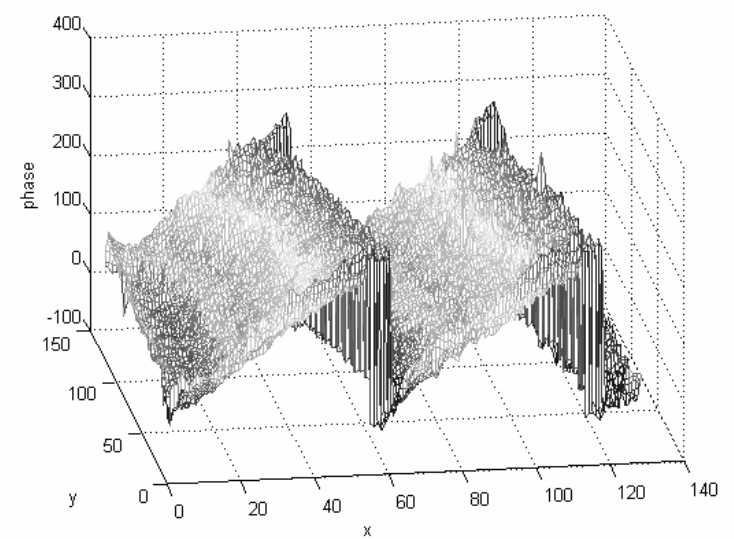

Figure 7: The (model phase + decoded error map), which is obtained finally by the codingdecoding process. For this example, the overall compression ratio was set to $2: 1$.

In Figure 5, we illustrate the smoothed model obtained using our technique, while Figure 6 illustrates the error map coded by the DCT technique. Figure 7 illustrates the model phase map added to the decoded error map.

To obtain an idea of the power of the non parametric technique, we computed the mean of the absolute error between the overall decoded map and the original phase map. We compared this with the conventional SCT compression technique, for the same overall byte allocation. The experiment is repeated over two compression ratios, 2:1 and 4:1. We report the results in Table 1. Note that the model based method for 4:1 ratio even outperforms the conventional method at 2:1 compression ratio.

Table 1: Table illustrating the mean absolute error (in radians) at each pixel location using the model based method described in the paper and the conventional method of compression.

\begin{tabular}{|l|r|r|}
\hline & Model based & Conventional \\
\hline $2: 1$ & 0.123 & 0.276 \\
\hline $4: 1$ & 0.156 & 0.346 \\
\hline
\end{tabular}

\section{Experimental Results: Unwrapping}

One of the purposes of smoothing the phase data using the non parametric technique is to aid the unwrapping functionality for digital elevation model computation. We unwrapped the model map (using a simplistic algorithm which is not described within the scope of this work) in Figure 5 and obtained a continuous map illustrated in Figure 8. The unwrapping of the phase map obtained by adding the model phase to the DCT compressed error also has the nice properties of continuity (Figure 9). However, the original map is not continuous, while unwrapped, ash shown in Figure 10.

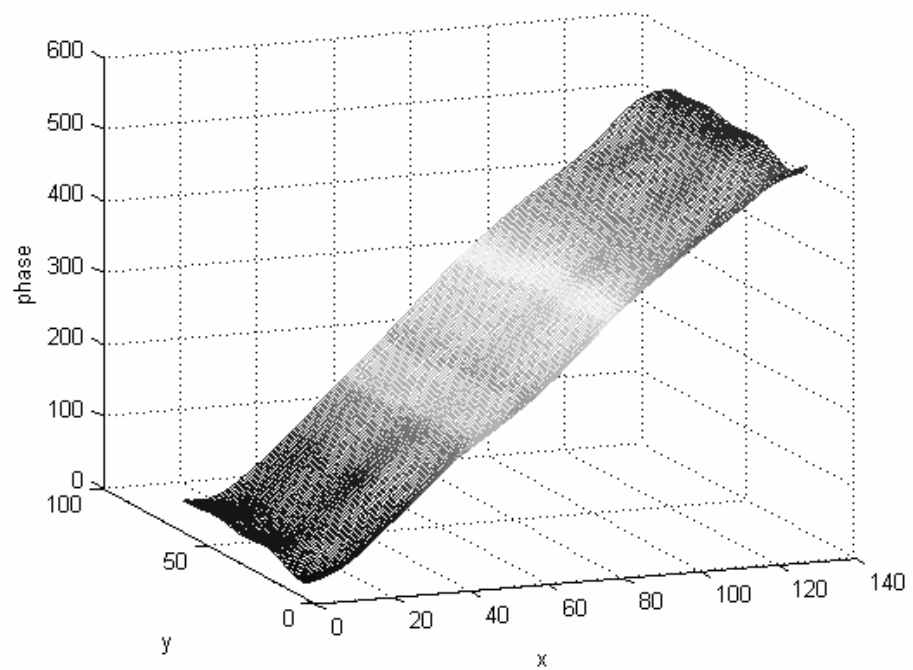

Figure 8: Unwrapping of the modeled phase map (note that 256 represents $2 \pi$ ).

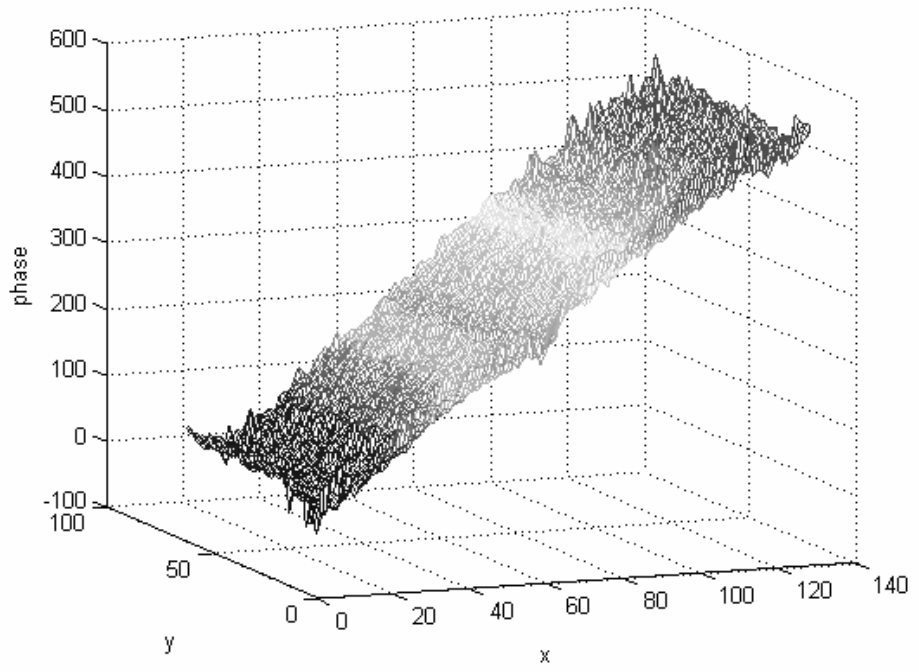


Figure 9: Unwrapping of the (modeled phase map +decoded error map).

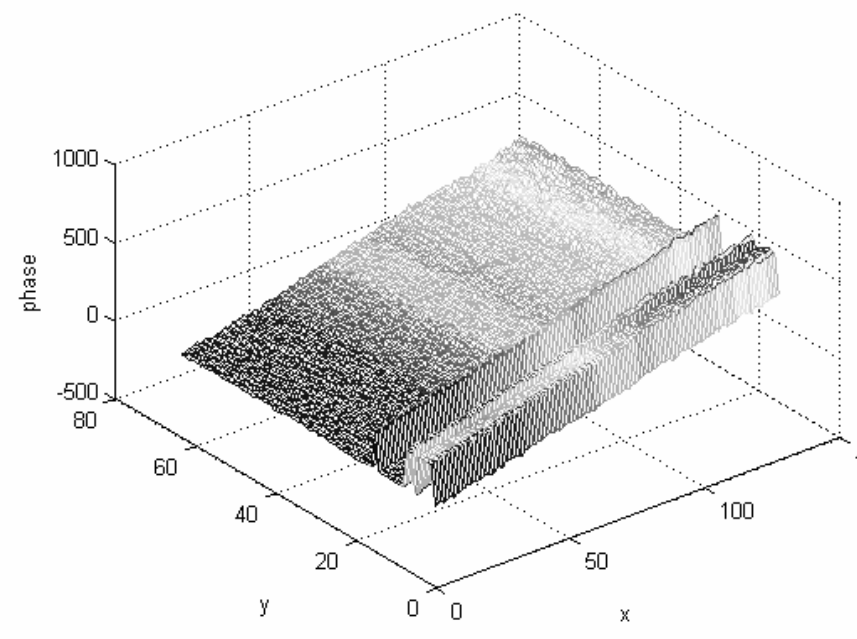

Figure 10: Unwrapping of the original map (shows discontinuities).

\section{Conclusions}

In this paper, we presented a model based technique for representing SAR phase images. The method can be used for filtering noise to aid unwrapping, as well as SAR image compression. This technique can be applied in all computer vision applications that involves modeling and analysis of vector fields.

\section{References}

[1] G. Franceschetti and G. Fornaro, "SAR interferometry," in Synthetic Aperture Radar Processing, G. Franceschetti and R. Lanari, Eds., chapter 4, pp. 167-223, CRC Press, New York, NY, USA, 1999.

[2] P. A. Rosen, S. Hensley, I. R. Joughin, et al., "Synthetic aperture radar interferometry," Proc. IEEE, vol. 88, no. 3, pp. 333-382, 2000.

[3] R. M. Goldstein, H. A. Zebker, and C. L. Werner, "Satellite radar interferometry: twodimensional phase unwrapping," Radio Science, vol. 23, no. 4, pp. 713-720, 1988.

[4] B. Aiazzi, S. Baronti, M. Bianchini, A. Mori, L. Alparone, "Filtering of Interferometric SAR Phase
Images as a Fuzzy Matching-Pursuit Blind Estimation, “JASP(2005), No. 20, 2005, pp. 32203230 .

[5] D. Maltoni, D. Maio, A.K. Jain, S. Prabhakar, Handbook of Fingerprint Recognition Springer, Chapter 3, New York, 2003

[6] W. Ying, J. Hu and D. Phillips, "A Fingerprint Orientation Model Based on 2D Fourier Expansion (FOMFE) and Its Application to Singular-Point Detection and Fingerprint Indexing, “ IEEE Trans. Pattern Analysis and 15Machine Intelligence, vol. 29, no. 4, pp. 573-585, April 2007.

[7] K. Sengupta and P. Burman, "A Curve fitting Problem and Its Application in Modeling Objects from Images," IEEE Trans. Pattern Analysis and Machine Intelligence, vol. 24, no. 5, pp. 674-686, 2002.

[8] www-star.stanford.edu/sar_group/snaphu/ 